\title{
Unicystic Ameloblastoma With Spindle Cell Differentiation
}

\author{
Rachana $^{1}$, Nandan S R K ${ }^{2}$, Aruna kumari $\mathrm{M}^{3}$
}

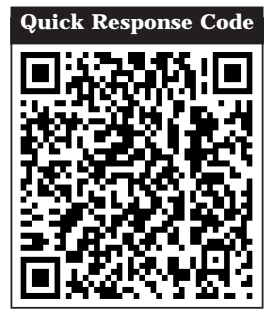

doi: $10.5866 / 2016.8 .10060$

${ }^{1}$ Post graduate

${ }^{2} \mathrm{H}$ ead of the Department

3Senior lecturer

Department of Oral Pathology \& Microbiology,

Kamineni Institute of Dental Sciences, Narketpally,

Nalgonda Dist. India

\section{Article Info:}

Received: J anuary 6, 2016

Review Completed: February 8, 2016

Accepted: March 7, 2016

Available Online: May, 2016 (www.nacd.in)

(c) NAD, 2016 - All rights reserved

\section{Email for correspondence:}

rachana.1114@gmail.com

\section{NTRODUCTION}

Amel oblastoma is one of the most common types of odontogenic tumor, however, it accounts for only $1 \%$ of all oral tumors. It is a benign tumor whose importance lies in its potential to grow to enormous size resulting in bone deformity. The origin of the tumor is thought to be from sources that include residual epithelium of the tooth-forming apparatus, such as the epithelial cell rests of Malassez; epithelium of odontogenic cysts; basal cells of the surface epithelium; epithelium of the enamel organ; and heterotopic epithelium from extraoral sites, such as the pituitary gland. The unicystic type comprises $10 \%-15 \%$ of all ameloblastomas. The

\begin{abstract}
:
The term unicystic ameloblastoma (UA) refers to those cystic lesions that show clinical, radiographic, or gross features of a jaw cyst, but on histologic examination show a typical ameloblastomatous epithelium lining part of the cyst cavity, with or without luminal and/or mural tumor growth. Although the histology suggests that cystic ameloblastomas follow a biol ogically low-grade course, recent evidencesuggests that they may often behave clinically as aggressive tumors by its high incidence of cortical perforation, tooth resorption, increase in lesion size, bony destruction, and a high rate of recurrence after simple enucleation. Here, we present a case report on unicystic variant of ameloblastoma in the mandible. It is important to remember that a proper and timely diagnosis of the character and extent of a $\cup A$, with a thorough histopathologic examination of the entire specimen, can help in the overall long-term wellbeing of the patient.
\end{abstract}

Key words: Unicystic ameloblastoma, spindle cells. 


\section{CASE REPORT}

A 40-year-old male reported to the Department of Oral Pathology and Microbiology, Kamineni institute of Dental sciences, Narketpally, with a complaint of swelling over the left lower half of face for past two and half months. On extraoral examination, buccally a diffuse swelling of about 5 $x 3 \mathrm{~cm}$ in size extending posteriorly from $1 \mathrm{~cm}$ away from the midline to $4 \mathrm{~cm}$ away from the angle of the mandible was seen (Figure 1). I ntraoral examination revealed diffuse swelling of size about $3 \times 4 \mathrm{~cm}$ extending from distal aspect of 32 to the mesial aspect of 36, lingually about $1.5 \times 1.5 \mathrm{~cm}$ swelling irt 35, which is non-tender and hard in consistency (Figure 2). 3D reformatted CT image revealed a unilocular radiolucency in the left mandibular region and thinning of lower border of mandible (Figure 3).

A clinical diagnosis of ameloblastoma and keratocystic odontogenic tumor was made. An incisional biopsy was advised and specimen submitted for histopathological examination. Microscopically hematoxylin and eosin stained section showed a bit of non keratinizing stratified squamous epithelium. The connective tissue stroma shows presence of areas of plump spindle shaped cells with mild nuclear and cellular pleomorphism al ong with nuclear hyperchromatism. It al so reveals few multinucleated gaint cells amidst fibro collagenous stroma and LS, TS of muscle tissue. Areas of hyalinised material surrounded by plump fibroblast, reactive new bone formation and chronic inflammatory cell infiltration is also evident. Based on the above features, a diagnosis of spindle cell tumor was made. For exact typing of the nature of the lesion, immunohistochemistry was performed with the markers Pancytokeratin, Vimentin, Desmin and Smooth muscle actin.

An excisional biopsy was advised and specimen submitted for histopathological examination. Microscopically hematoxylin and eosin stained section showed cystic lining of variable thickness with prominent hyperchromatic palisading basal layer, along with proliferation of lining in form of plexiform strands (Figure 4). Numerous engorged and dilated blood vessels are seen within the lining. Some areas of cystic lining epithelium shows dysplastic features like nuclear hyperchromatism and altered nuclear cytoplasmic ratio. Other section also reveal ed proliferation of cystic lining into a wall of connective tissue in form of large follicular and plexiform network with interconnecting strands. The peripheral cells of these follicles and strands exhibit dysplastic features such as nuclear hyperchromatism, altered nuclear cytoplasmic ratio and hyperplasia of these basal cells, whereas centre of thesefollicles show more cellular area rather than loose stellate like cells. Connective tissue capsule exhibits presence of numerous spindle shaped cells exhibiting nuclear hyperchromatism and pleomorphism (figure 5). There is also evidence of proliferation of endothelial cells and numerous small and large engorged blood vessels. The connective tissue also shows presence of dense bundles of collagen fibres, moderate inflammatory infiltrate chiefly composed of lymphocytes and plasma cells. Other deeper areas of connective tissue show muscle tissue and peripheral bone. Based on the above features a diagnosis of unicystic ameloblastoma with dysplasia and spindle cell differentiation is made. For exact typing of the tumor and nature of spindle cells immunohistochemistry with pancytokeratin (Figure 6) and vimentin are made.

\begin{tabular}{|l|c|c|}
\hline MARKER & $\begin{array}{c}\text { EPITHELIAL } \\
\text { ISLANDS }\end{array}$ & STROMA \\
\hline Cytokeratin & $4+$ & Negative \\
\hline Vimentin & Negative & $3+$ \\
\hline SMA & Negative & $2+$ \\
\hline
\end{tabular}

\section{DISCUSSION}

Odontogenic tumors are a large group of neoplasm or hamartomatous growth of the jaws arising from tooth forming tissues. ${ }^{1}$ Among all odontogenic tumors ameloblastoma is the most significant one. ${ }^{2}$ Ameloblastoma is the second most common odontogenic neoplasm, and only odontoma outnumbers it in reported frequency of occurrence. ${ }^{3}$ As per the WHO system of 2003, ameloblastoma is classified based on differences in biologic behavior, treatment plan and recurrence rate as follows:

(1) classic solid/multicystic ameloblastoma,

(2) unicystic ameloblastoma,

(3) peripheral ameloblastoma,

(4) desmoplastic ameloblastoma, including the so-called hybrid lesions.

$\mathrm{UA}$ is a rare type of ameloblastoma accounting for approximately $6 \%$ of all ameloblastoma. The lesion in essence is a well defined cystic sac lined by 


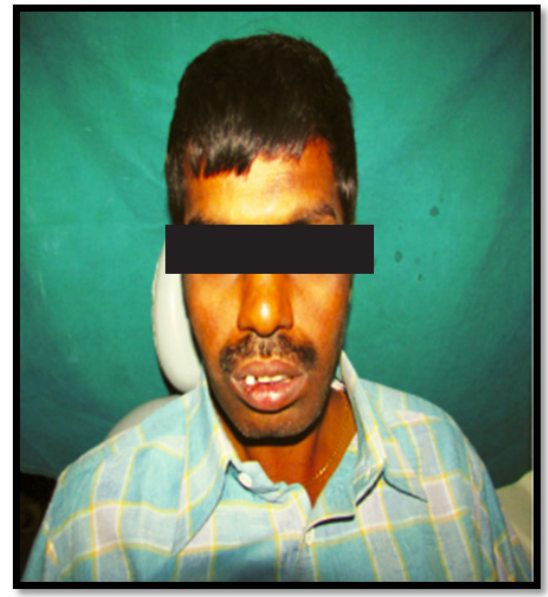

Figure 1: Clinical picture showing diffuse swelling in the left lower posterior region of jaw.

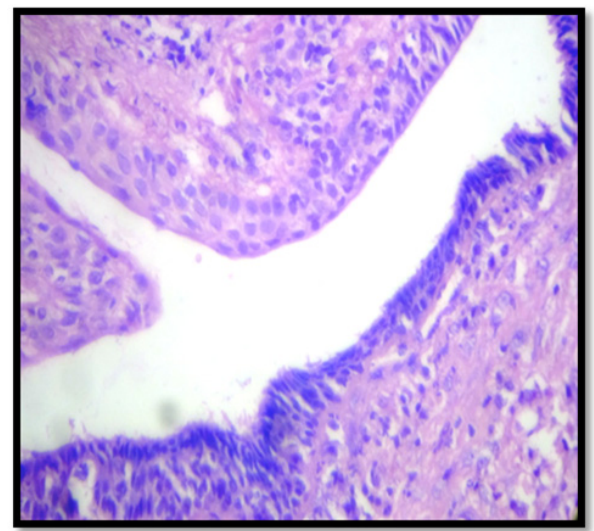

Figure 4: $H$ \& E stained section showing prominent hyperchromatic palisading basal layer (10X).

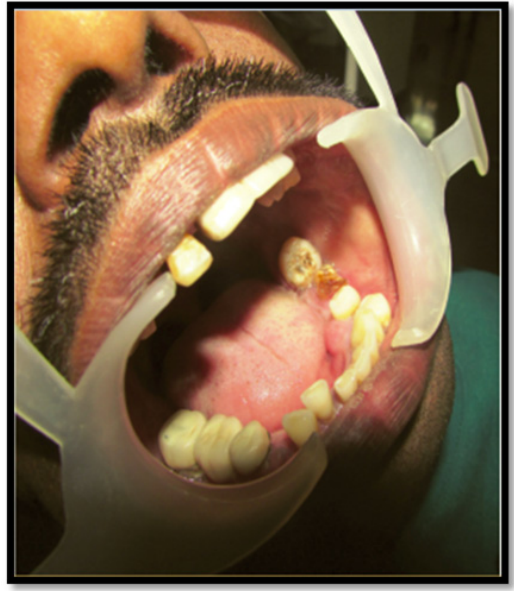

Figure 2: Clinical picture showing swelling extending from distal aspect of 32 to the mesial aspect of 36

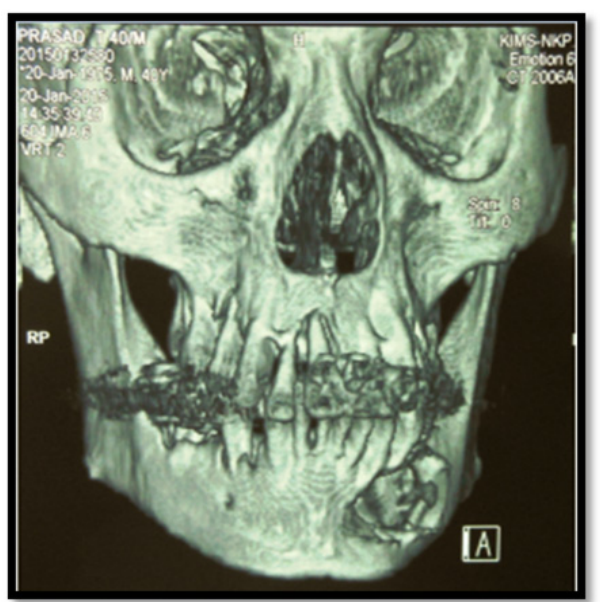

Figure 3: 3D reformatted CT image showing unilocular radiolucency in the left mandibular region and thinning of lower border of mandible

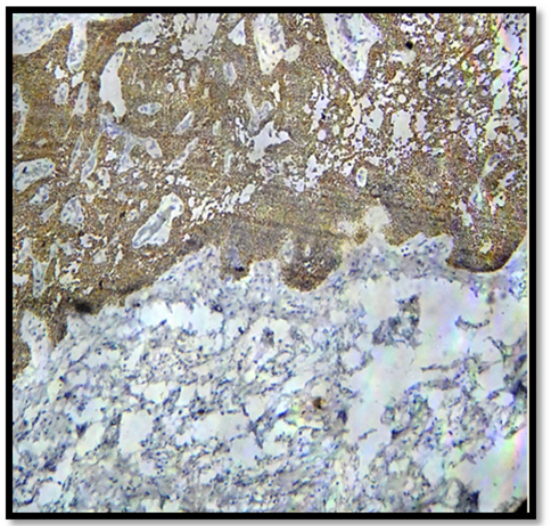

Figure 3: Figure 6: Cytokeratin positivity is seen in epithelial islands
Figure 5: H \& E stained section showing numerous spindle shaped cells exhibiting nuclear

hyperchromatism and pleomorphism in the connective tissue capsule

(40X). ameloblastomatous epithelium with or without luminal and or mural proliferation. Most of UA are associated with impacted teeth and such lesions are referred to as dentigerous variants and thoselesions which are not in association with an impacted tooth are called non dentigerous type. ${ }^{4}$ The concept of unicystic ameloblastoma was first introduced by Robinson and Martinez in 1977 and later this was variously termed as mural, monocystic, intracystic, cystogenic, or cystic ameloblastoma. ${ }^{5}$ Ackermann classified this entity into three histologic groups

1) Luminal UA (tumor confined to the luminal surface of the cyst)
2) Intraluminal/plexiform UA (nodular proliferation intothelumen without infiltration of tumor cells into connective tissue wall), and

3) Mural UA (invasive islands of ameloblastomatous epithelium in the connective tissue wall not involving the entire epithelium).

o Type $3 a$ - Islands not connected to cyst lining

o Type $3 b$ - Island connected to cyst lining. ${ }^{6}$

Leider et al. (1985) proposed three pathogenic mechanisms for the evolution of UA. 
(1) The reduced enamel epithelium which is associated with a developing tooth undergoes ameloblastic transformation with subsequent cystic development.

(2) Ameloblastomas arise in dentigerous cysts or in others in which the neoplastic ameloblastic epithelium is preceded temporarily by a nonneoplastic stratified squamous epithelial lining.

(3) A solid ameloblastoma undergoes cystic degeneration of the ameloblastic islands, with subsequent fusion of multiple microcysts and develops into unicystic lesions. ${ }^{2}$

Still the pathogenesis of cystic ameloblastoma is not clear. The reason why some ameloblastomas become completely cystic may be related to epithelial dysadhesion (e.g. defective desmosomes) or, more likely to the intrinsic production of proteinases, enzymes that normally degrade the central zone of enamel organ after tooth development.' In 1977, Robinson showed that biological behavior of UA arising within the wall of an antecedent cystic lesion of the jaw is different from that of the conventional solid and multicystic ameloblastoma. Vickers and Gorlin conducted a detailed study to find out the early ameloblastic changes that occur within the wall of an odontogenic cyst. According to them, to justify the diagnosis of ameloblastoma in a cyst of the jaw following three features should be present together:

(1) Hyperchromatism of the basal cell nuclei of the epithelium lining the cystic cavity.

(2) Palisading of the basal cells and polarization of the nuclei of the basal cells lining the cystic cavity, (i. e., situated at the distal ends of the cells)

(3) Cytoplasmic vacuolation of these basal cells. ${ }^{8}$

Dysplastic ameloblastic cells with spindle-cell transformation were seen arising from a cystic lining with features of a unicystic ameloblastoma. Occurrence of atypia may take the form of cellular pleomorphism of the basaloid cells, large polygonal squamoid cells, with pale vesicular nuclei, increased and abnormal mitotic figures, presence of granular cells and clear cells, and spindle-shaped de- differentiation. Spindle-shaped cells in a malignant lesion can be characterized as malignant fibroblasts (MF) or cancer-associated fibroblasts (CAF). Alpha smooth muscle actin (alpha-SMA), fibroblastactivated protein (FAP), fibroblasts-specific-protein1 (FSP-1/S100A4), PDGF-beta, and neuron-glial antigen (NG2) are most common markers currently used. Spindle cell is characterized as stromal myofibroblast if it is positive to alpha-SMA and may show positivity for Vimentin or Desmin. Spindle cell proliferation may be not of fibroblastic orgin, could be a variant of dysplastic odontogenic tissue. ${ }^{9}$

\section{CONCLUSION}

Spindle-cell differentiation from an cysticlining of unicystic amel oblastoma is relatively rare and few reported cases do not convey adequate data on the biological behaviour of this lesion. More cases are need to be studied with respect to biological behaviour of this lesion.

\section{REFERRENCES}

1. Anil Singh, Shoyeb Shaikh, Fahad M. Samadi, Shikha Shrivastava. Maxillary unicystic ameloblastoma: A review of the literature. Natl J Maxillofac Surg 2011; 2(2):163-8.

2. J ayavirsinh J Barad, Neelampari Parikh. Unicystic Ameloblastoma of Mandible with Mural Proliferation: A Case Report. J Res Adv Dent 2015; 4:1:23-7.

3. Vasant Shewale, Ashish Deshmukh, Rakesh Sorathia. Unicystic Ameloblastoma: A Case Report. IJ HSR 2014; 4(7):313-6.

4. Vikram Deshmukh, Raunak Sunil Patwa, Prachi Bhanudas Chhindam. An unusual case of bilateral unicystic amel oblastoma in third molar region. J Dent Allied Sci 2014; 3(2):99-101.

5. Ankita Tandon. Malignant Squamous Changes in Unicystic Ameloblastoma: A Case Report and Review of Literature. J interdiscipl Med Dent Science 2014, 2:4.

6. Arpita Rai, Monica Arora, Venkatesh G Naikmasur. Unicystic Plexiform Aameloblastoma. Univ Res] Dent 2015; 5(3):211-5.

7. Shamimul Hasan, Jamia Millia Islamia. Unicystic Ameloblastoma of Mandible: A case report and review of literature. Arch Dent 2012; 3(1):25-8.

8. Vikrant $\mathrm{O}$ Kasat, Freny R Karjodkar, Ruchi Ladda. Plexiform unicystic amel oblastoma -a case report and review of literature. J Health Res 2014; 1(2):103-6.

9. Venkatesh V. Kamath, Krishnanand Satelur, Komali Yerlagudda. Spindle cell variant of amel oblastic carcinoma arising from an unicystic amelobastoma: Report of a rare case. Dent Res J 2012; 9(3):328-33. 